\title{
DNA damage response and MCL-1 destruction initiate apoptosis in adenovirus-infected cells
}

\author{
Andrea Cuconati, ${ }^{1,2,4}$ Chandreyee Mukherjee, ${ }^{1,2}$ Denise Perez, ${ }^{2}$ and Eileen White ${ }^{1-3,5}$ \\ ${ }^{1}$ Howard Hughes Medical Institute, ${ }^{2}$ Center for Advanced Biotechnology and Medicine, ${ }^{3}$ Department of Molecular Biology \\ and Biochemistry, Cancer Institute of New Jersey, Rutgers University, Piscataway, New Jersey 08854, USA
}

Expression of adenovirus E1A deregulates cell proliferation to facilitate viral DNA replication, prompting the initiation of apoptosis signaled primarily through proapoptotic BAK in productively infected cells. We demonstrate here that in uninfected cells, BAK is complexed with the anti-apoptotic BCL-2 family member Myeloid Cell Leukemia 1 (MCL-1). E1A expression during infection resulted in the specific down-regulation of MCL-1 through destabilization of the protein and loss of the mRNA. Upon loss of the MCL-1-BAK complex, BAK complexed with either BAX in proapoptotic E1B mutant adenovirus-infected cells, or with the adenovirus BCL-2 homolog E1B 19K in cells infected with the wild-type virus in which apoptosis is inhibited. Loss of MCL-1 was required to initiate the apoptotic pathway in infected cells as restoration of MCL-1 expression rescued infected cells from E1A-induced apoptosis. Analogous to E1A expression, DNA damage down-regulates MCL-1, and adenovirus infection resulted in the accumulation of phosphorylated H2AX and ataxia-telangiectasia mutant protein (ATM), hallmarks of DNA double-strand breaks. Thus, MCL-1 may function by maintaining BAK in an inactive state, and the loss of MCL-1 upon activation of the DNA damage response, perhaps through replication stress induced in virus infected cells, may be required to initiate the apoptotic response.

[Keywords: Apoptosis, MCL-1, E1B 19K, adenovirus, DNA damage, BAK]

Received September 30, 2003; revised version accepted October 22, 2003.

Cells infected by adenovirus are prompted out of quiescence and into the S-phase-like state by expression of the E1A proteins, which antagonize the function of the p300 family of coactivators and the retinoblastoma protein (RB; White 2001). By interfering with p300 and RB, which activates E2F-1, E1A deregulates cell-cycle control and stimulates apoptosis. As premature death of infected cells is detrimental to virus replication, DNA viruses have evolved an assortment of countermeasures to inhibit apoptosis, and in the case of adenovirus, E1B 19K fulfills this function (White 2001; Cuconati and White 2002).

E1B 19K shares sequence and functional homology with the BCL-2 family of cellular prosurvival proteins, and is a powerful inhibitor of apoptosis induced by a wide variety of stimuli (White 2001; Cuconati and White 2002). In cells infected with an adenovirus mutant lacking the E1B 19K gene, E1A expression results in the activation of apoptosis manifested by degradation of ge-

${ }^{4}$ Present address: Discovery Research, ViroPharma Incorporated, Exton, PA 19341, USA.

${ }^{5}$ Corresponding author.

E-MAIL ewhite@cabm.rutgers.edu; FAX (732) 235-5795.

Article published online ahead of print. Article and publication date are at http://www.genesdev.org/cgi/doi/10.1101/gad.1156903. nomic and viral DNA and premature cell death, which limits virus replication (White 2001; Cuconati et al. 2002). In transformed cells, E1A expression induces the stabilization and accumulation of the p53 tumor suppressor protein (Lowe and Ruley 1993), and E1A-mediated apoptosis is exclusively dependent on p53 (Debbas and White 1993; Lowe et al. 1994; Degenhardt et al. 2002). However, the mechanism by which E1A induces apoptosis in infected cells is not well defined. Although E1A expression during productive infection also induces p53 accumulation, apoptosis induction appears to be independent of E1A's ability to stabilize p53 (Chiou and White 1997), as the viral E1B 55K-E4orf6 complex eliminates p53 by targeting it for degradation in proteasomes (Querido et al. 1997, 2001; Steegenga et al. 1998). Thus, E1A expression induces p53 accumulation, which normally induces growth arrest and apoptosis in response to cellular stresses such as DNA damage, and although the virus encodes a mechanism to disable this $\mathrm{p} 53$ response, apoptosis still occurs by a p53-independent pathway in infected cells. This may have necessitated that the virus also encode the E1B 19K function to inhibit this p53independent apoptotic pathway.

Clues to how apoptosis is activated by E1A in infected cells came from elucidation of the mechanism by which 
the E1B 19K protein blocks apoptosis. Yeast two-hybrid screening identified proapoptotic BAK and BAX as E1B $19 \mathrm{~K}$-interacting proteins, and E1B 19K expression blocks cell death induced by BAK or BAX overexpression (Farrow et al. 1995; Han et al. 1996). The E1B 19K protein interacts with and inhibits the endogenous activated forms of proapoptotic BAK and BAX (Perez and White 2000; Sundararajan and White 2001; Sundararajan et al. 2001; Cuconati et al. 2002) that are required for releasing apoptogenic mitochondrial proteins (cytochrome and Smac/DIABLO, among others), which serve to activate cysteine proteases of the caspase family that implement apoptosis (Lindsten et al. 2000; Wei et al. 2001; Zong et al. 2001; Degenhardt et al. 2002). BAK and BAX activation can occur through direct interaction with proapoptotic BH3-only proteins such as tBID, and/or through inactivation of pro-survival BCL-2 family members. Activation of BAK and BAK is thought to occur through changes in their protein conformation, followed by oligomerization into a high-molecular weight protein complex that is associated with compromised mitochondrial membrane permeability (Korsmeyer et al. 2000; Cory and Adams 2002). For example, during tumor necrosis factor (TNF)- $\alpha$-mediated apoptosis, caspase- 8 activation by the receptor complex and cleavage of BID to truncated tBID propagates death signaling to mitochondria. In adenovirus infected cells, tBID interacts with and activates both BAK and BAX, and the E1B $19 \mathrm{~K}$ protein binds to activated $\mathrm{BAK}$ and $\mathrm{BAX}$ and prevents their co-oligomerization. This E1B 19K-BAK and E1B 19K-BAX interaction prevents the release of proapoptotic mitochondrial proteins, thereby inhibiting caspase- 9 and caspase- 3 activation and apoptosis (Perez and White 2000; Sundararajan and White 2001; Sundararajan et al. 2001). The interaction of E1B $19 \mathrm{~K}$ with BAK and BAX is sufficient to block TNF- $\alpha$-mediated apoptosis as BAK and BAX-deficient cells are resistant to TNF- $\alpha$ (Degenhardt et al. 2002). Because BAK and BAX are general effectors of apoptotic signaling, their inhibition by E1B 19K could account for its function, not only in apoptosis mediated by TNF- $\alpha$, but also by E1A.

E1A-mediated apoptosis in cells infected with an E1B $19 \mathrm{~K}$ mutant adenovirus similarly results in BAK and BAX activation, co-oligomerization, mitochondrial release, caspase activation, and apoptosis (Cuconati et al. 2002). In cells infected with the wild-type virus, in which apoptosis is blocked by E1B $19 \mathrm{~K}$ expression, BAK activation still occurs, but the interaction of E1B $19 \mathrm{~K}$ with activated BAK prevents activation of BAX, co-oligomerization of BAK and BAX, mitochondrial protein release, caspase activation, and apoptosis (Cuconati et al. 2002). Thus, signaling of apoptosis by E1A in adenovirusinfected cells primarily goes through BAK, which is targeted by the E1B $19 \mathrm{~K}$ protein. However, deficiency in both BAK and BAX is required to block apoptosis in cells infected with an E1B 19K mutant adenovirus, suggesting that in the absence of BAK, there is likely a salvage pathway for BAX activation and apoptosis induction $(\mathrm{Cu}-$ conati et al. 2002). This varies from TNF- $\alpha$ death signaling, in which tBID activates both BAK and BAX and E1B
$19 \mathrm{~K}$ interacts with both. There is no caspase- 8 activation or conversion of BID to tBID during E1A-mediated apoptosis in adenovirus-infected cells, suggesting that the mechanism of BAK activation by E1A was distinct from that signaled by TNF- $\alpha$ (Perez and White 2000; Cuconati et al. 2002). Nonetheless, BAK emerged as the key mediator of E1A-mediated apoptosis during productive virus infection, and we began a search for the mechanism of BAK activation.

As BAK (and BAX) activation can occur through induction of and interaction with proapoptotic $\mathrm{BH} 3$-only proteins, or through the loss of function and interaction with anti-apoptotic BCL-2 family members, infected cells were screened for changes in the levels of these proteins and for their interaction with BAK. We report here that BAK interacted with anti-apoptotic BCL-2 family member MCL-1 in healthy, uninfected cells. Adenovirus infection and E1A expression repressed MCL-1 expression, which was associated with the induction of BAK-BAX complex formation and apoptosis that was blocked by restoring MCL-1 levels. In infected cells in which apoptosis was blocked by E1B 19K expression, MCL-1 down-regulation still occurred, but E1B 19K interacted with $\mathrm{BAK}$, and $\mathrm{BAK}-\mathrm{BAX}$ interaction was prevented. As MCL-1 down-regulation initiates the apoptotic response to ultraviolet and $\gamma$ irradiation (Nijhawan et al. 2003), we examined infected cells for induction of a DNA-damage response. Adenovirus infection induced phosphorylation of H2AX and ATM, even when apoptosis was inhibited by E1B 19K expression in cells infected with wild-type virus. Thus, E1A expression may trigger a DNA-damage response, resulting in not only p53 stabilization, but also in MCL-1 down-regulation, which then serves as a priming event for BAK activation and apoptosis. This may necessitate that adenovirus encode a BAK and BAX inhibitor in addition to a p53 antagonist, and explain the means by which E1A expression sensitizes cells to DNA damage-mediated apoptosis.

\section{Results}

Interaction of BAK with MCL-1 is lost in adenovirus-infected cells

BAK and BAX undergo stepwise conformational changes revealed by epitope exposure that are induced by E1A expression during infection with a proapoptotic E1B 19K mutant adenovirus. Specifically, an amino-terminal BAK epitope is exposed, whereas BAX exhibits exposure of amino- and carboxy-terminal epitopes. Conformational change of BAX but not BAK, and oligomerization of BAK and BAX are prevented by expression of E1B 19K in wildtype virus-infected cells, and interaction of E1B 19K was observed predominantly with BAK rather than BAX $(\mathrm{Cu}-$ conati et al. 2002). These observations suggest that E1A expression signals a conformational change in BAK and the formation of a proapoptotic complex between BAK and BAX, causing a conformational change in BAX that is prevented by E1B $19 \mathrm{~K}-\mathrm{BAK}$ interaction. The nature of the proapoptotic signal responsible for BAK activation by 
E1A led us to search for BAK-interacting proteins in vivo.

Because $\mathrm{BAK}$ and $\mathrm{BAX}$ are regulated through activation by $\mathrm{BH} 3$-only proteins, or are sequestered by antiapoptotic BCL-2 family members, we screened BAK for interaction with both classes of apoptotic regulators. HeLa cells were either mock infected, infected with wild-type (Ad5d1309) adenovirus, or with an E1B $19 \mathrm{~K}$ gene-deleted adenovirus (Ad5d1337), and cells were harvested $24 \mathrm{~h}$ post-infection. Immunoprecipitations were carried out using antibodies directed to a variety of known prosurvival (BCL-2, MCL-1, and BCL-X ${ }_{\mathrm{L}}$, and proapoptotic $\mathrm{BH} 3$-only proteins (BIM, BID, and tBID), using two different antibodies directed against BAK. Multiple antibodies were utilized for immunoprecipitations, as recognition of BCL-2 family members varies with epitope availability that is greatly influenced by protein conformation and/or protein-protein interactions. Among the antibodies tested, an anti-MCL-1 antibody (MCL-1) robustly coimmunoprecipitated BAK with MCL-1 from mock-infected cells, but not from Ad5d1309 or Ad5dl337-infected cells, whereas a second antiMCL-1 antibody (MCL-1 Ab-1) did so weakly (Fig. 1). Conversely, an antibody directed against BAK (BAK-TM) robustly coimmunoprecipitated MCL-1 with BAK in mock-infected, but not Ad5d1309 or Ad5dl337 infected cells, whereas a second anti-BAK antibody BAK(Ab-1) did so weakly (Fig. 1). A third anti-BAK antibody (BAKNT) also coimmunoprecipitated MCL-1 when either used directly for immunoprecipitation or when crosslinked to sepharose prior to immunoprecipitation (data not shown). E1B 19K coimmunoprecipitated with BAK from Ad5d1309-infected cells (Fig. 1) as expected (Cuconati et al. 2002). These observations indicated that BAK interacted with MCL-1 in uninfected cells, which was supplanted by an interaction between E1B 19K and BAK upon infection. In Ad5dl337 infected cells, in which apoptosis was induced, BAK coimmunoprecipitated with $\mathrm{BAX}$, and no $\mathrm{BAK}-\mathrm{BAX}$ coprecipitation was observed in mock or Ad5d1309-infected cells (Fig. 1) as expected (Cuconati et al. 2002). No coimmunoprecipitation of BAK with BCL-2, BCL- $\mathrm{X}_{\mathrm{L}}, \mathrm{BIM}, \mathrm{BID}$, or tBID was observed in uninfected or infected cells (data not shown). These results suggested that MCL-1 may sequester BAK in an inactive form in healthy, uninfected cells, and that E1B 19K may functionally substitute for MCL-1 in cells infected with the wild-type virus.

\section{Down-regulation of MCL-1 protein levels in adenovirus-infected cells}

The apparent modest reduction in MCL-1 levels in infected compared with mock-infected cells at $24 \mathrm{~h}$ postinfection (Fig. 1) led us to examine MCL-1 expression during a time course of infection as a possible mechanism behind the loss of the MCL-1-BAK interaction. HeLa cells were mock infected, or infected with the Ad5d1309, Ad5d1337, the E1B deletion mutant Ad5E1B ${ }^{-}$, the $E 1 A$ deletion mutant Ad5E1A ${ }^{-}$, and the $E 1 A+E 1 B$ deletion mutant Pac3. Samples were collected at 0,12 , $24,36,48$, and $72 \mathrm{~h}$ post-infection and subjected to Western blot analysis for MCL-1, BCL-2, BCL- $\mathrm{x}_{\mathrm{L}}$, and actin. Levels of MCL-1 were found to decrease starting 12-24 h post-infection in Ad5d1309, Ad5d1337, and Ad5E1B ${ }^{-}$infected cells, culminating in the substantial loss of MCL-1 protein (Fig. 2A). In contrast, levels of MCL-1 were unaffected in mock, Ad5E1A ${ }^{-}$, and Pac3-infected cells (Fig. 2A). Furthermore, the loss of MCL-1 occurred in Ad5dl309-infected cells, in which apoptosis was inhibited, indicating that it was not merely a consequence of cell death. Levels of BCL-2, actin (Fig. 2A), and BCL- $\mathrm{X}_{\mathrm{L}}$ (data not shown) did not change under any conditions, suggesting that the loss of MCL-1 protein was specific and occurred through an E1A-dependent mechanism. These observations suggest that the abrogation of MCL1-BAK interaction may be prompted by a loss of MCL-1 protein levels.

\section{Destabilization of $M C L-1$ protein in adenovirus-infected cells}

We investigated the cause for the loss of MCL-1 protein levels by assessing the stability of the MCL-1 protein during adenovirus infection of HeLa cells. Cells were either mock infected or infected with Ad5d1309 or Ad $5 d 1337$ for $24 \mathrm{~h}$, and then treated with a combination of the proteasome inhibitor epoxomicin (EPO) and the
Figure 1. BAK and MCL-1 form a complex that is disrupted during adenovirus infection. Immunoprecipitation (IP) of BAK and MCL-1 from mock, Ad5dl309, Ad5d1337 infected cells was carried out with anti-p19, anti-PCNA, anti-BAK(Ab-1), anti-BAK(TM), anti-MCL1, anti-MCL-1(Ab-1) antibodies (Abs) from the soluble fraction of cells lysed in CHAPS-containing buffer at 24 $\mathrm{h}$ post-infection. Mock-infected HeLa cell lysate (M) was utilized as a marker for MCL-1, BAK, and BAX expression levels. Western blotting was carried out on precipitated material with an anti-MCL-1 antibody, anti-BAK plus anti-BAX antibodies, or an anti-E1B 19K antibody. Samples of lysates collected before IP (Lysates) were analyzed to ascertain total protein levels prior to immunoprecipitation.

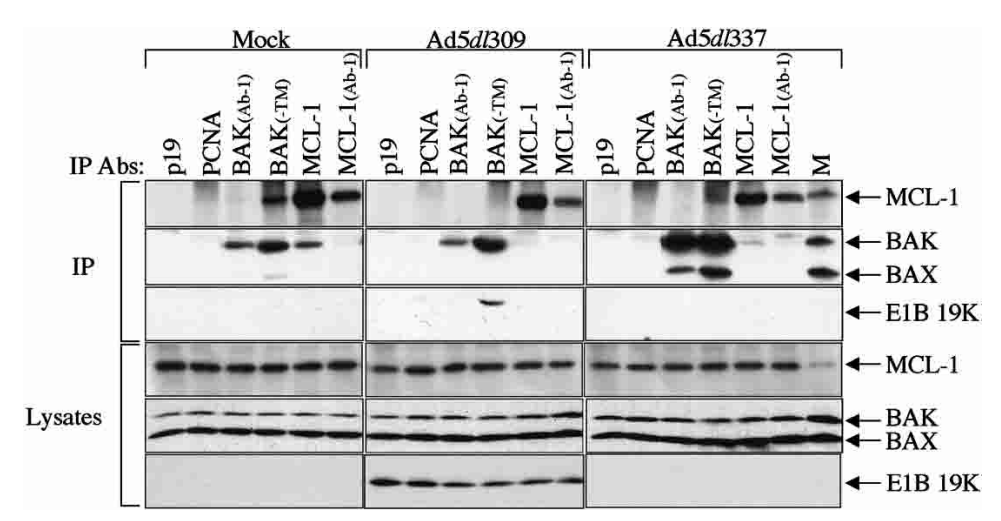




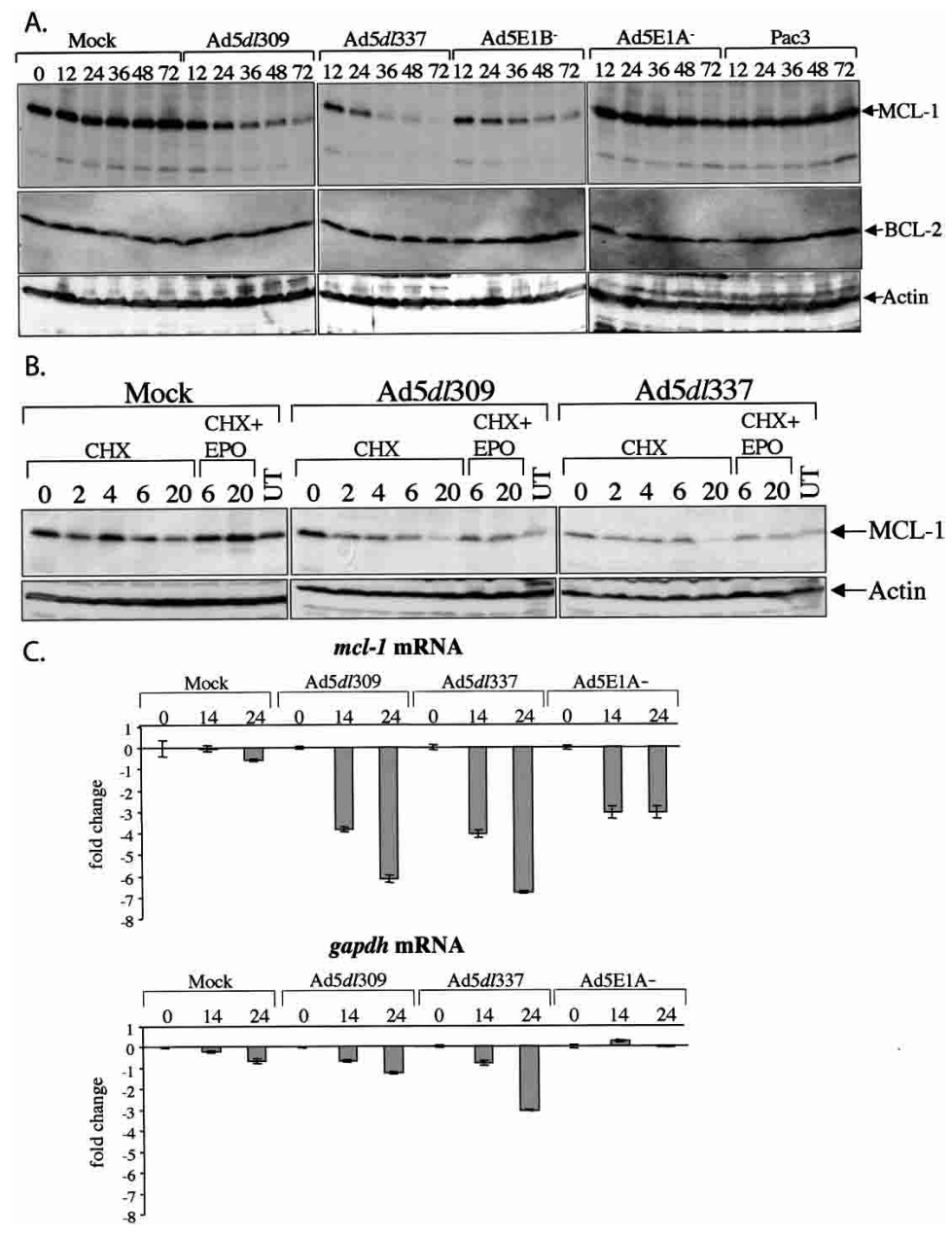

Figure 2. E1A expression during adenovirus infection causes the loss of MCL-1 protein. (A) HeLa cells were mock, Ad5d1309, Ad5d1337, Ad5E1B ${ }^{-}$, Ad5E1A', or Pac3 infected, and representative samples were collected at 0 (for mock only), 12, 24, 36, 48, and $72 \mathrm{~h}$ post-infection, and analyzed by Western blotting with antibodies against MCL-1, BCL-2, and actin. (B) Adenovirus infection stimulates proteasome-mediated turnover of MCL-1. HeLa cells were mock, Ad5d1309, or Ad5dl337 infected for $24 \mathrm{~h}$, and then treated with cycloheximide (CHX) for 0, 2, 4, 6, and $20 \mathrm{~h}$, or a combination of CHX plus epoxomicin (EPO) for 6 or $20 \mathrm{~h}$, or were left untreated (UT). Samples were collected at the indicated time points and analyzed by Western blotting with anti-MCL-1 and anti-actin antibodies. $(C)$ The effect of adenovirus infection on mcl-1 mRNA levels. Total RNA from mock, Ad5d1309, Ad5d1337, or Ad5E1Ainfected cells was isolated at 0,14 , and 24 h post-infection, and was analyzed by Taqman real-time PCR using a probe and primers specific for mcl-1 mRNA. Fold change represents the difference in fluorescence signal in the 14- and 24-h samples compared with the corresponding 0-h sample. Error bars, the standard deviation of triplicate samples. Samples were also analyzed by Taqman real-time PCR with primers and a probe specific for gapdh. translation inhibitor cycloheximide (CHX) for 6 or $20 \mathrm{~h}$, with CHX alone for $0,2,4,6$, or $20 \mathrm{~h}$, or were left untreated (UT). Inhibition of new protein synthesis under CHX treatment reduced the levels of MCL-1 protein modestly in mock-infected cells, which was more pronounced in adenovirus-infected cells (Fig. 2B). Addition of EPO for either 6 or $20 \mathrm{~h}$ prevented much of the loss of MCL-1 protein in both infected and uninfected cells, indicating that MCL-1 protein turnover is regulated by ubiquitin-dependent proteasome degradation. In mockinfected cells, MCL-1 was relatively stable with an apparent half-life $>4 \mathrm{~h}$. In contrast, adenovirus infection dramatically increases the rate of MCL-1 protein turnover with an apparent half-life of $<2 \mathrm{~h}$, which is reversed by addition of a proteasome inhibitor. Levels of actin remained constant under all conditions. These observations indicated that adenovirus infection stimulates the specific turnover of MCL-1, suggesting a mechanism for initiation of cell death during adenovirus infection that is upstream of E1B $19 \mathrm{~K}$ inhibition.

\section{Reduction of intracellular levels of mcl-1 mRNA during adenovirus infection}

We ascertained the effect of infection on mcl-1 mRNA levels through real time PCR analysis of total RNA from either mock, Ad5d1309, Ad5d1337, or Ad5E1 A- infected HeLa cells at 0,14 , and $24 \mathrm{~h}$ post-infection. In infected cells expressing E1A, loss of mcl-1 mRNA is progressive over the course of infection, exhibiting up to sixfold repression (Fig. 2C). In contrast, in Ad5E1 A ${ }^{-}$infected cells, loss of the mcl-1 message does not progress beyond $14 \mathrm{~h}$ post-infection, and levels do not decrease by more than threefold. Levels of gapdh mRNA also decreased during infection, but significantly less than mcl-1 mRNA (Fig. $2 \mathrm{C})$. The data suggest that there is an E1A-specific mechanism for the repression of mcl-1 expression during infection, which could lead to reduced MCL-1 protein levels, and disruption of the BAK-MCL-1 complex.

\section{Restoration of MCL-1 expression rescued apoptosis induced by adenovirus infection}

To determine whether MCL-1 is functionally capable of rescuing infected cells from adenovirus-induced apoptosis, we utilized transient transfection to overexpress MCL-1 in infected HeLa cells. Cells were transfected with plamids encoding MCL-1L fused to a FLAG epitope (FLAG-MCL-1L), E1B 19K fused to a V5 epitope (E1B $19 \mathrm{~K}-\mathrm{V} 5)$, and $\beta$-galactosidase fused to an Xpress epitope ( $\beta$-gal-Xpress). All three sets of transfected cells were 
Cuconati et al.

mock, Ad5d1309, or Ad5dl337-infected at 24 h posttransfection. Cells were fixed $36 \mathrm{~h}$ post-infection on glass coverslips, stained for immunofluorescence with antibodies directed to the cognate epitope tag, and then stained with Hoescht's dye for determination of chromatin condensation. The percentage of cells undergoing apoptosis (exhibiting condensed chromatin) in the transfected population was determined visually by counting the number of condensation-positive cells in 100-300 transfected cells per sample. As depicted in Figure 3, A and $\mathrm{B}$, cells exhibiting condensed chromatin were largely seen in the Ad5d1337-infected sample. Of those that were positive for transfection, the $\beta$-gal-Xpress-transfected sample under Ad5d1337 infection was found to contain $\sim 40 \%$ apoptotic cells, as compared with $<2 \%$ and $1 \%$ apoptotic cells in the Ad5dl337-infected E1B 19K-V5 and FLAG-MCL-1 expressing cells, respectively (Fig. 3B). As expected, Ad5d1309 infection did not cause measurable levels of apoptosis, due to expression of E1B $19 \mathrm{~K}$, and introduction of E1B $19 \mathrm{~K}$ into cells infected with Ad5d1337 rescued the cells from apoptosis (Fig. 3B). Interestingly, the introduction of MCL-1 into Ad5d1337infected cells blocked apoptosis similarly to E1B 19K, clearly indicating that MCL-1 overexpression was able to complement E1B 19K deletion. These observations showed that MCL-1 was functionally capable of inhibiting adenovirus-induced apoptosis, and suggest that under normal conditions, endogenous MCL-1 must be lost in order for adenovirus-induced apoptosis to occur.

\section{MCL-1 down-regulation is not sufficient for BAK activation}

Adenovirus infection and E1A expression results in exposure of an amino-terminal epitope on BAK revealed by enhanced immunoprecipitation by BAK(Ab-1) (Fig. 1; Cuconati et al. 2002). To test whether MCL-1 downregulation is sufficient for exposure of this BAK epitope indicative of conformational activation of BAK, we utilized RNA interference to down-regulate MCL-1. Despite significant reduction of MCL-1 by transfection with one or two siRNAs specific for $m c l-1$, no increase in BAK immunoprecipitation was observed with BAK/Ab1) or two other conformation-sensitive anti-BAK antibodies (Fig. 3C), nor was there any apparent affect on cell viability (data not shown). BAK-MCL-1 coimmunoprecipitation was observed with the BAK-TM antibody as expected (Fig. 3C). Thus, MCL-1 down-regulation by E1A may be required for apoptosis, but it is not sufficient for BAK activation and cell death.

Induction of H2AX and ATM phosphorylation by E1A expression during adenovirus infection

The E1A-dependent down-regulation of MCL-1 observed during adenovirus infection was strikingly similar to the mechanism of apoptosis induction in response to DNA damage. Ultraviolet or $\gamma$ irradiation stimulate the elimination of MCL-1 protein expression, which is required,
Figure 3. Exogenous MCL-1 overexpression rescues apoptosis in adenovirus-infected cells. (A) MCL-1 prevents apoptosis-associated chromatin condensation in Ad5dl337-infected cells. HeLa cells expressing FLAGMCL-1, E1B 19K-V5, and $\beta$-Gal-Xpress by transfection were mock, Ad5dl309, or Ad5dl337 infected for $30 \mathrm{~h}$, and analyzed by immunofluorescence staining and Hoescht's dye DNA staining for chromatin condensation. Rhoadmine (red) stain represents cells expressing the transfected gene, whereas blue stain represents chromatin staining. White arrows indicate the corresponding chromatin-stained cells from the matching rhodamine-stained micrograph. (B) MCL-1 expression rescues infected cells from adenovirus-induced apoptosis in the absence of E1B 19K. The percentage of transfected mock, Ad5d1309, or Ad5d1337 infected cells exhibiting chromatin condensation by Hoescht's dye staining was determined by counting only those staining positive for the transfected gene. Error bars, the standard deviation of counts from two separate experiments. $(C)$ Down-regulation of MCL-1 is not sufficient for BAK activation. HeLa cells were transfected with either control or mcl-1-specific siRNAs $(1)$ or $(1+2)$. Levels of MCL-1 and BAK were determined by Western blotting $($ top $)$ and BAK activation was determined by IP with BAK antibodies (bottom). Enhanced BAK (Ab-1) immunoprecipitation indicative of BAK activation occurs in apoptotic cells (see Fig. 1). BAK-MCL-1 coimmunoprecipitation with the BAK-TM antibody is observed in the MCL-1 Western blot of the BAK immunoprecipitations (bottom).

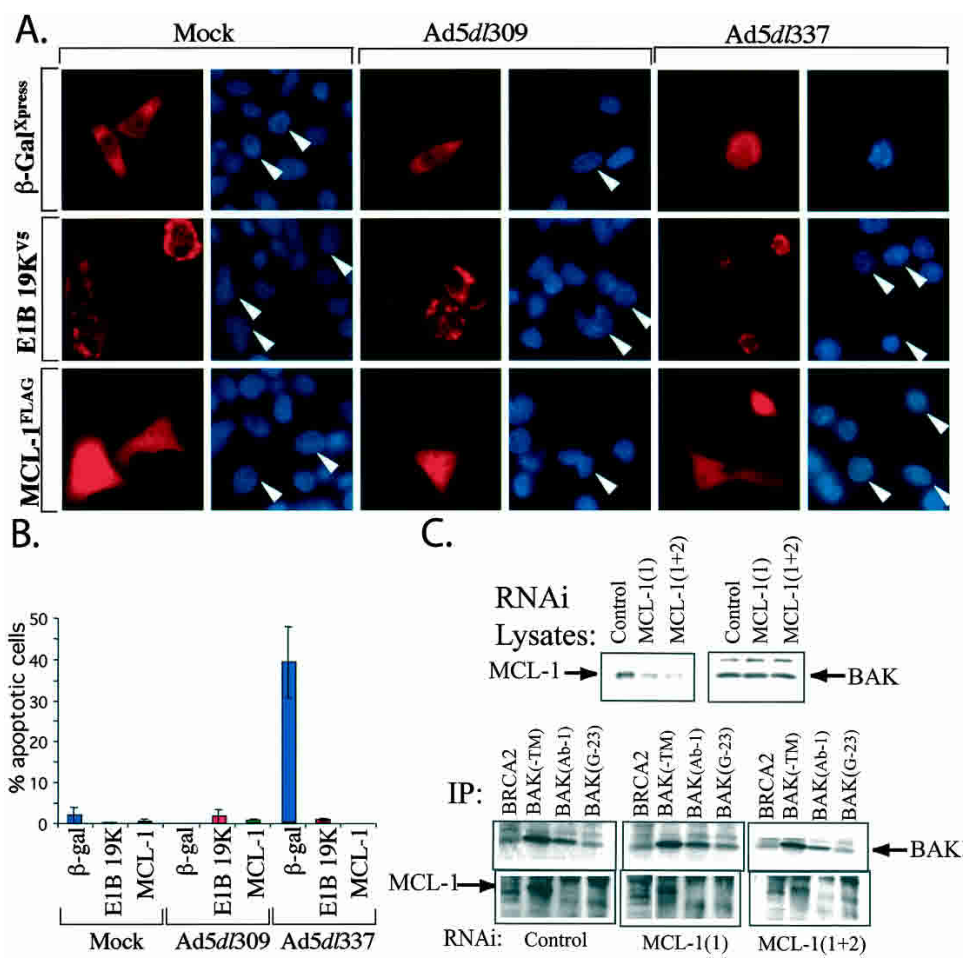


but not sufficient, for apoptosis (Nijhawan et al. 2003). Indication of induction of a DNA-damage response in adenovirus-infected cells was explored by testing for accumulation of the phosphorylated forms of ATM and H2AX. ATM responds to double-strand DNA breaks by undergoing autophosphorylation at serine 1981, and a downstream substrate in the ATM pathway, H2AX, becomes phosphorylated on serine 139. Antibodies specific for the phosphorylated forms of ATM (ATM1981S-P) and $\mathrm{H} 2 \mathrm{AX}(\gamma \mathrm{H} 2 \mathrm{AX})$ were utilized in Western blots, IP-Westerns, and indirect immunofluorescence to determine whether their levels were induced by adenovirus infection. Whereas levels of $\gamma \mathrm{H} 2 \mathrm{AX}$ were low as expected in uninfected cells, the levels accumulated dramatically in cells infected with the wild-type virus (Ad5d1309; Fig. 4A). $\gamma \mathrm{H} 2 \mathrm{AX}$ levels also accumulated in cells infected with the two E1B mutant viruses (Ad5d1337 and $\left.\mathrm{Ad} 5 \mathrm{E} 1 \mathrm{~B}^{-}\right)$, however, this was expected, as these viruses induce apoptosis, the DNA fragmentation of which creates double-strand DNA breaks. Interestingly, infection with the two E1A deletion mutants $\left(\mathrm{E}^{-} \mathrm{A}^{-}\right.$and Pac3) did not induce $\gamma \mathrm{H} 2 \mathrm{AX}$ accumulation (Fig. 4A), indicating the requirement for E1A expression. E1A and E1B 19K were expressed only by the viruses that encoded their genes, and cellular actin levels were comparable among the samples as an indication of equal protein leading (Fig. 4A).

To test for ATM activation by phosphorylation upon infection, ATM was immunoprecipitated from mock, Ad5d1309, or Ad5d1337-infected cells and subjected to Western blotting using either ATM or and ATM phospho-serine 1981-specific antibodies. Only Ad5d1309 and Ad5d1337, but not mock-infected cells, displayed evidence of ATM phosphorylation on serine 1981 (Fig. 4B). Double-label indirect immunofluorescence for $\gamma \mathrm{H} 2 \mathrm{AX}$, and the adenovirus $72 \mathrm{kD}$ DBP as a marker for infected cells, revealed similar pronounced induction of serine 139 phosphorylated H2AX (Fig. 4C). Indirect immunoflourescence with the phospho-serine 1981 ATM antibody also demonstrated its induction upon infection with both the wild-type Ad5d1309 and proapoptotic Ad5d1337 adenoviruses (Fig. 4C). This suggested that cells responded to E1A expression during virus infection in a manner that resembled the cellular response to DNA damage, not only with respect to p53 induction, but also evidenced by accumulation of phosphorylated forms of H2AX and ATM, which may be linked to the loss of MCL-1, which facilitates apoptosis.

\section{Discussion}

\section{MCL-1 regulates adenovirus-induced apoptosis}

The mitochondrial checkpoint, regulated by proapoptotic BCL-2 family members BAK and BAX, plays an important role in the regulation of death signaling prompted by E1A expression in adenovirus-infected cells. Specifically, E1A expression in E1B 19K mutant virus-infected cells induces conformational changes in BAK and BAX, and the formation of a high-molecular

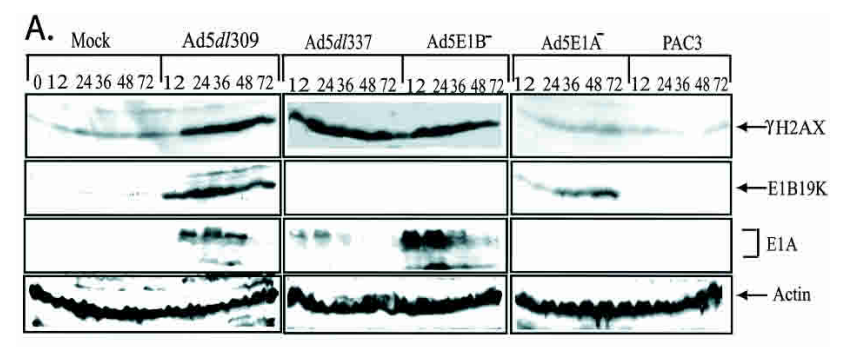

B.
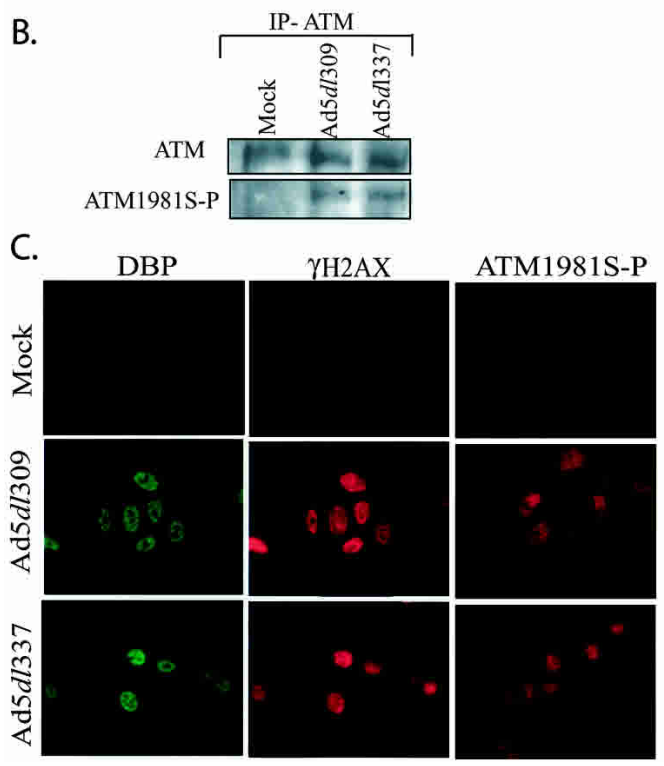

Figure 4. Induction of $\mathrm{H} 2 \mathrm{AX}$ and $\mathrm{ATM}$ phosphorylation during virus infection. (A) HeLa cells were mock, Ad5dl309, Ad5d1337, Ad5E1B-, Ad5E1A-, or Pac3 infected, and representative samples were collected at 0 (for mock only), 12, 24, 36, 48, and $72 \mathrm{~h}$ post-infection (see Fig. 2) and analyzed by Western blotting with antibodies against $\gamma \mathrm{H} 2 \mathrm{AX}$ (phospho-serine 139specific form of H2AX), E1B 19K, E1A, and actin. (B) ATM was immunoprecipitated from Mock, Ad5dl309, and Ad5dl337 infected HeLa cells (MOI 100 at $24 \mathrm{~h}$ post-infection) and was Western blotted for either ATM or ATM1981S-P (phospho-serine 1981-specific form of ATM), as indicated. (C) Mock, Ad5d1309, and Ad5dl337 infected HeLa cells (MOI 100 at $24 \mathrm{~h}$ post-infection) were fixed and stained for indirect immunoflourescence with antibodies directed against the adenovirus 72 $\mathrm{kD}$ DBP, $\gamma \mathrm{H} 2 \mathrm{AX}$ (double-label indirect immunflourescence), and ATM1981S-P, as indicated.

weight BAK-BAX protein complex, which coincides with the release of cytochrome $\mathrm{c}$ from the mitochondria, caspase- 9 and caspase- 3 activation and cell death. In the presence of E1B $19 \mathrm{~K}$ in cells infected with the wild-type virus, BAK displays a change in conformation, which is associated with interaction of BAK with the E1B 19K protein, but conformational changes in BAX and the formation of the BAK-BAX complex are abrogated. This interruption of apoptosis signaling through mitochondria is manifested by failure to release cytochrome $\mathrm{c}$ and activate caspase- 9 and caspase-3, which extends the viability of infected cells.

Proof of the requirement for BAK and BAX in E1Amediated apoptosis of adenovirus-infected cells was re- 
vealed when BAK and BAX deficiency prevented apoptosis induction upon infection with and E1B 19K mutant adenovirus, and greatly enhanced virus production $(\mathrm{Cu}-$ conati et al. 2002). Furthermore, this validated BAK and BAX as the functional targets for E1B 19K in inhibition of apoptosis during productive adenovirus infection. Thus, BAK and BAX function as part of an antiviral response to limit virus replication through apoptosis induction, which is thwarted by E1B 19K-BAK and E1B 19-BAX interaction to sustain the viability of infected cells until the replication cycle is complete. We expected that identification of the signal by which infection and E1A expression activates BAK upstream of E1B 19K should provide insight into the virus-host cell interactions that initiate the apoptotic defense cascade. BAK is normally sequestered by antiapoptotic MCL-1, and what is likely a DNA damage response to virus infection represses MCL-1 expression, priming infected cells to apoptosis induction.

BAK has been reported recently to interact with the mitochondrial outer-membrane protein VDAC2, which acts to restrict BAK activation (Cheng et al. 2003). There may be multiple BAK protein complexes that respond to different death stimuli to regulate BAK activation and apoptosis. In addition to MCL-1 and VDAC2, BAK also interacts with E1B $19 \mathrm{~K}$ and $\mathrm{BBID}$, and BAK protein interactions may be required for both BAK activation and inhibition.

In healthy, uninfected cells, 24-kD BAK migrates with a native molecular weight of at least $60-70 \mathrm{kD}$ by gel filtration chromatography, suggesting that it may be complexed with other cellular proteins (Sundararajan et al. 2001). In contrast, BAX appears predominantly monomeric, fractionating with an expected native molecular weight of $\sim 21 \mathrm{kD}$ (Sundararajan and White 2001; Sundararajan et al. 2001). The interaction of BAK with 42-kD MCL-1 reported here may explain the aberrant native molecular weight of BAK, and suggests that MCL-1 may be a BAK chaperone to regulate the proapoptotic activity of BAK. MCL-1 fractionates by gel-filtration chromatography with a native molecular weight of at least $60 \mathrm{kD}$ with significant overlap with the BAK fractionation profile consistent with BAK-MCL-1 complex formation as well as complex formation with other proteins (R. Sundararajan and E. White, unpubl.). MCL-1 has potent antiapoptotic function (Kozopas et al. 1993; Reynolds et al. 1994; Craig 2002), and has been reported to interact with BAK in the yeast two-hybrid system (Bae et al. 2000). As shown here, MCL-1 may function in mammalian cells by interacting with and inhibiting BAK, and loss of MCL-1 upon E1A expression in adenovirus-infected cells may enable the apoptotic response (Fig. 5A). Restoration of MCL-1 is sufficient to block apoptosis induced by E1A expression in adenovirus-infected cells, and the E1B 19K protein apparently functionally substitutes for the absence of MCL-1 by interacting with and inhibiting BAK once the MCL-1-BAK complex is disrupted (Fig. 5B). In that way, E1B 19K can be thought of as a functional homolog of MCL-1 more so that BCL-2, which may predominantly function through interaction with and inhibition of $\mathrm{BH}$-only proteins rather than BAK and BAX (Cheng et al. 2001).

\section{E1A provokes a DNA damage response that results in MCL-1 elimination}

The elimination of MCL-1 by E1A expression during adenovirus infection occurred by both increased ubiquitindependent turnover of the protein and repression of expression of the mRNA. The expression of MCL-1 and its prosurvival function is regulated by growth factors and is essential for early mammalian development (Craig 2002). Proapoptotic stimuli are also known to repress MCL-1 expression. E2F-1 activation, for example, represses mcl-1 expression, and E1A activates E2F-1 through binding and inhibition of RB (Croxton et al. 2002a,b). DNA damage, induced by either ultraviolet or $\gamma$ irradiation, down-regulates MCL-1 protein levels in all, or in part, by stimulating proteasome-mediated turnover of MCL-1 protein (Nijhawan et al. 2003). Although E1Amediated, E2F-1 activation could account for down-regulation of the mcl-1 mRNA, the enhanced turnover of the

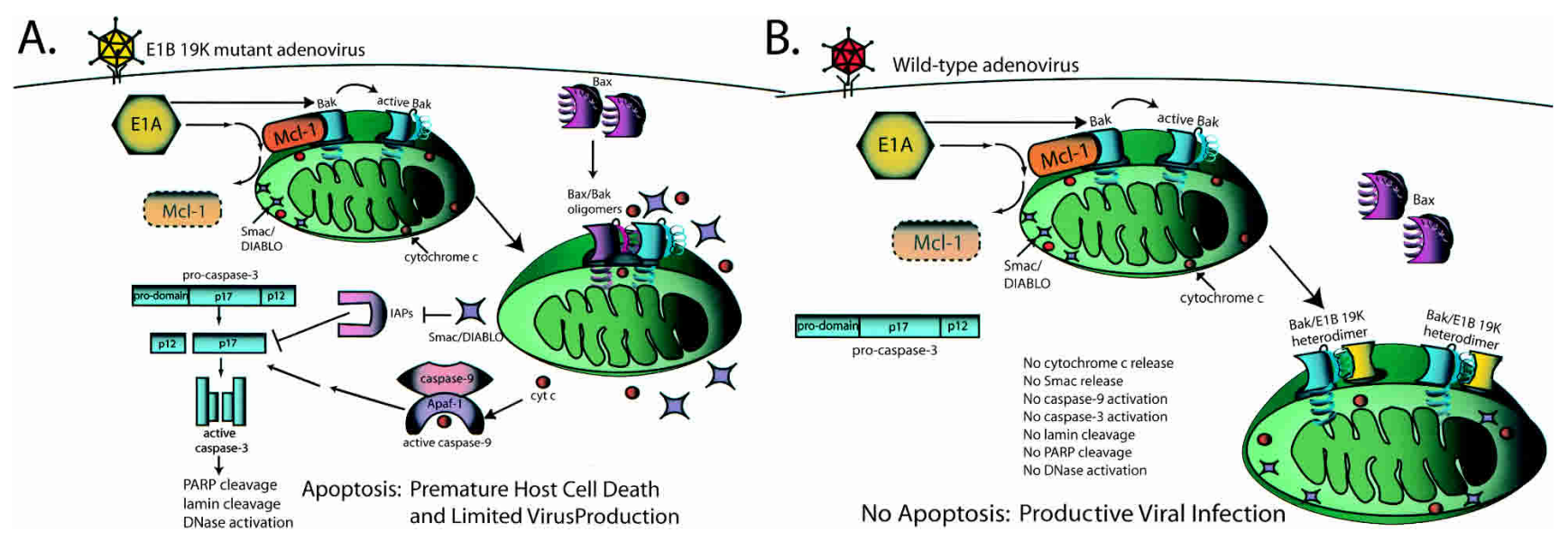

Figure 5. Schematic representation of the regulation of adenovirus-induced apoptosis in infected cells. See text for explanation. 
MCl-1 protein in adenovirus-infected cells resembled a DNA-damage response. Remarkably, H2AX, a known substrate of the PI3 kinases ATM and ATR that initiate the cellular response to DNA damage (Rogakou et al. 1998, 2000; Ward and Chen 2001; Redon et al. 2002; Bakkenist and Kastan 2003), and ATM itself, become dramatically phosphorylated upon adenovirus infection (Fig. 4). The induction of $\gamma \mathrm{H} 2 \mathrm{AX}$ (the phosphorylated form of H2AX) and ATM 1981S-P occurred in both wildtype virus-infected cells, in which apoptosis is blocked by E1B 19K expression, and in cells infected with a proapoptotic E1B 19K mutant virus. Thus, induction of $\gamma \mathrm{H} 2 \mathrm{AX}$ and ATM 1981S-P upon infection occurred upstream of apoptosis induction.

E1A expression also resembles a DNA-damage response by inducing p53 accumulation (Lowe and Ruley 1993), which is seen during productive adenovirus infection as long as the E1B 55K gene is deleted (Chiou and White 1997). In infected HeLa cells, p53 induction by E1A maps primarily to the p300 and not the RB-binding region (Chiou and White 1997), and E1A inhibits the polyubiqitination of $\mathrm{p} 53$ by $\mathrm{p} 300$, which promotes, along with MDM-2, p53 degradation in proteasomes/Grossman et al. 2003). It is not known whether regulation of p53 turnover by p300 is responsible p53 accumulation in response to DNA damage. Likely, a combination of events initiated by E1A expression signals a cellular stress response that primes cells for apoptosis. As we can only correlate activation of a DNA-damage response by E1A to initiation of apoptosis, it will be of great interest to interfere with upstream components of the DNAdamage signaling (ATM and ATR, for example) to determine whether they are necessary or sufficient for apoptosis induced by E1A.

Although adenovirus encodes within E1B 19K and E1B $55 \mathrm{~K}$ mechanisms to disable this cellular response to stress, the upstream pathways to activate p53, ATM, and $\mathrm{H} 2 \mathrm{AX}$ in response to E1A expression appear intact. How adenovirus infection and E1A expression induces a DNA-damage response is not clear, but could be explained by the induction of double-strand DNA breaks resulting from either replication stress mediated by deregulation of cell cycle control by E1A, or by viral DNAreplication intermediates that may be perceived as damaged DNA, or by changes in chromatin structure that are known to activate ATM (Bakkenist and Kastan 2003). When expressed in transformed cells, the human papilloma virus E7 gene product, which functions analogously to E1A by antagonizing RB and deregulating cell cycle control, also induces $\gamma \mathrm{H} 2 \mathrm{AX}$ that is associated with genomic instability (Duensing and Munger 2002). $\mathrm{H} 2 \mathrm{AX}$ is required to prevent genomic instability and tumorigenesis in vivo (Bassing et al. 2003; Celeste et al. 2003). This raises the possibility that transformation by viral oncogenes may initiate a DNA-damage response triggering down-regulation of MCL-1 and susceptibility to apoptosis. E1A expression sensitizes cells to apoptosis induced by DNA-damaging agents (Sanchez-Prieto et al. 1996; Brader et al. 1997; Shao et al. 1997; Stiewe et al. 2000; Viniegra et al. 2002), as does specific knock-down of MCL-1 (Nijhawan et al. 2003). This priming of apoptosis by E1A may require adenovirus to encode an antiapoptotic function to sustain viability during oncogenesis.

Finally, although elimination of MCL-1 is required for apoptosis in response to DNA damage, it is not sufficient (Nijhawan et al. 2003). Eliminating MCL-1 may render BAK susceptible to activation by $\mathrm{BH} 3$-only proteins that are coordinately induced in infected cells. Interestingly, the antiapoptotic latent membrane protein-1 of EpsteinBarr virus induces MCL-1 accumulation (Wang et al. 1996). Taken together, MCL-1 may be part of an antiviral response to render infected cells susceptible to apoptosis. This may have necessitated the evolution of viral countermeasures to either prevent the destruction of MCL-1, or for the viruses themselves to encode functional substitutes of MCL-1 to maintain viability of infected cells until the replication cycle is complete. It will be of great interest to determine whether other classes of viruses possess similar activities.

\section{Materials and methods}

\section{Virus infection and Western blotting}

HeLa cells were mock infected, or infected with adenovirus type 5 (Ad5) wild-type Ad5dl309 (Jones and Shenk 1979), the E1B $19 \mathrm{~K}$ gene deletion mutant Ad5 $d 1337$ (Pilder et al. 1984), the $E 1 B$

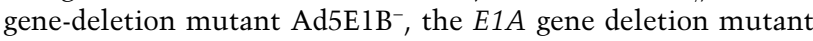
Ad5E1A', or the $E 1 A$ and $E 1 B$ gene-deletion mutant Pac3 (Chiou et al. 1994), at a multiplicity of infection (MOI) of 100 by standard methods (White et al. 1984). Attached and floating cells were harvested at 0 (for mock only), 12, 24, 36, 48, and 72 $h$ post-infection by scraping and centrifugation. All cell pellets were resuspended in $2 \times$ Laemmli buffer and vortexed briefly. Each lysate sample was subjected to $17 \%$ SDS-PAGE, and analyzed by Western blotting (Perez and White 1998) with the following primary antibodies: anti-MCL-1 rabbit polyclonal (Stressgen Biotechnologies); anti-BCL-2 hamster monoclonal polyclonal (PharMingen); anti-E1A and anti-actin mouse monoclonal (Oncogene Research); anti-E1B 19K rabbit polyclonal (Sundararajan and White 2001); and anti- $\gamma \mathrm{H} 2 \mathrm{AX}$ (anti-H2AX phospho Ser 139) rabbit polyclonal (Upstate Biotechnology) antibodies. Blots were developed with horseradish peroxidase-conjugated secondary antibodies using the ECL system (Amersham-Pharmacia Biotech).

\section{Immunoprecipitation}

For each immunoprecipitation reaction, HeLa cells were infected as indicated and harvested at 24 and $48 \mathrm{~h}$ post-infection by scraping and lysed in 2.0\% 3-[(3-cholamidopropyl)-dimethylammonio]-1-propanesulfonate (CHAPS)-containing lysis buffer and immunoprecipitated as described previously (Perez and White 2000). Immunoprecipitations were carried out with the following primary antibodies: anti-p19 rabbit polyclonal and anti-PCNA mouse monoclonal (Santa Cruz Biotechnology); anti-BAK(Ab-1) mouse monoclonal (Oncogene Research); antiBAK (minus transmembrane domain, or -TM) rabbit polyclonal (PharMingen); anti-BAK-NT rabbit polyclonal (Upstate Biotechnology); anti-MCL-1 rabbit polyclonal (Stressgen); and antiMCL-1 mouse monoclonal (Oncogene Research); anti-MYC rabbit polyclonal (Santa Cruz Biotechnology); anti-BAX(11-30) rabbit polyclonal (Santa Cruz Biotechnology); anti-BAX (150-165) 
rabbit polyclonal (Oncogene Research); anti-BAX(43-61) rabbit polyclonal (PharMingen); and anti-BAK G-23 rabbit polyclonal (Santa Cruz Biotechnology). Immunoprecipitates were resuspended in $2 \times$ Laemmli buffer, and a sample of each immunoprecipitation reaction was subjected to $17 \%$ SDS-PAGE. Western blotting of immunoprecipitates was carried out with the following primary antibodies: anti-MCL-1 rabbit polyclonal (Stressgen), anti-BAX(11-30) rabbit polyclonal (Santa Cruz); anti-BAK(23-37) (Upstate Biotechnology); and anti-E1B 19K rabbit polyclonal (Sundararajan and White 2001) antibodies. The secondary antibody used was horseradish peroxidase-conjugated goat anti-rabbit (Southern Biotech). For immunoprecipitation of ATM, HeLa cells were Mock, Ad5d1309, or Ad5dl337 infected as described above, and cells were harvested and lysed in $50 \mathrm{mM}$ Tris (pH 7.5), $150 \mathrm{mM} \mathrm{NaCl}, 1 \%$ Tween-20, 0.05\% NP-40, 50 $\mathrm{mM} \mathrm{NaF}$, with protease inhibitors. Immunoprecipitations were carried out with an anti-ATM rabbit polyclonal antibody (Rockland Immunochemical Research) and subjected to $7.5 \%$ SDSPAGE, and Western blotted with either anti-ATM or antiATM1981S-P rabbit polyclonal antibodies (Rockland Immunochemical Research; Bakkenist and Kastan 2003).

\section{Determination of MCL-1 protein stability}

HeLa cells were infected as indicated at an MOI of 100 and treated with $30 \mu \mathrm{g} / \mathrm{mL}$ of cycloheximide (CHX, Sigma) solubilized in ethanol starting at $24 \mathrm{~h}$ post-infection for durations of 0 , $2,4,6$, or $20 \mathrm{~h}$, or CHX and $200 \mathrm{nM}$ of epoxomicin (EPO, Alexis) solubilized in dimethyl sulfoxide for durations of 6 or $20 \mathrm{~h}$, or mock treated with dimethyl sulfoxide and ethanol alone. Cells were harvested at the indicated time point by scraping and centrifugation, and were resuspended in $2 \times$ Laemmli buffer and vortexed briefly. Lysate samples were subjected to $17 \%$ SDSPAGE, and analyzed by Western blotting with the following primary antibodies: anti-MCL-1 rabbit polyclonal (Stressgen Biotechnologies) and anti-actin mouse monoclonal (Oncogene Research).

\section{Analysis by real-time PCR}

A total of $2 \times 10^{6} \mathrm{HeLa}$ cells per condition were infected as indicated at an MOI of 100, and were harvested by scraping and centrifugation at 0,14 , and $24 \mathrm{~h}$ post-infection. Total RNA was isolated through the use of the RNeasy Mini kit (QIAGEN), and 100 ng of total RNA from each condition was subjected to realtime RT-PCR with the Taqman EZ-RT PCR kit (PE Applied Biosystems) using recommended conditions, in the ABI Prism 7700 Sequence Detector. Optimal primer and probe sequences for mcl-1 real-time PCR were determined with the Primer Express software version 1.5 (Applied Biosystems), and were supplied compliments of Integrated DNA Technologies; probe sequence was supplied with $5^{\prime}$ linkage to the reporter dye 6-carboxy-fluorescein (FAM), and 3' linkage to Black Hole quencher (Integrated DNA technologies). MCL-1 primer sequences were 5'-TGAAATCGTTGTCTCGAGTGATG-3' and 5'-TCACAA TCGCCCCAGTTT-3'. MCL-1 probe sequence was 6FAM-5'TCCATGTTTCAGCGACGGCGTAA-3'- Black Hole. Primers and a probe for gapdh PCR were obtained from Applied Biosystems, and probe was supplied with $5^{\prime}$ linkage to the reporter dye 2,7-dimethoxy-4, 5-dichloro-6-carboxy-fluorescein (JOE), and 3' linkage to the quencher 6-carboxy-tetramethylrhodamine (TAMRA). Reactions were conducted in triplicate, and the fold change in fluorescence was determined by dividing the mean of the signal from the 14- and 24-h samples by the mean of the signal from the corresponding 0 -h samples, which was then nor- malized to 0. Standard deviation was calculated from the triplicate samples.

\section{Indirect immunofluorescence}

HeLa cells transfected by electroporation with plasmid pCDNA3-FLAG-MCL-1L (Bae et al. 2000; kindly provided by J. Bae and A.J. Hsueh, Stanford University), plasmid pCDNA3.1E1B-V5 (Kasof et al. 1998), or plasmid pCDNA3.1- $\beta$-Gal-Xpress (Invitrogen) were grown on glass coverslips and were infected with Ad5d1309, Ad5d1337, or were mock infected for $30 \mathrm{~h}$ at an MOI of 100 . Cells on coverslips were fixed in $4 \%$ paraformaldehyde, and indirect immunofluorescence was performed as described previously (Perez and White 1998), except that coverslips were blocked with $4 \%$ BSA/PBS for $1 \mathrm{~h}$ at $37^{\circ} \mathrm{C}$. Coverslips were stained with anti-FLAG mouse monoclonal (Sigma), V-5 mouse monoclonal (Invitrogen), anti-Xpress mouse monoclonal (Invitrogen), anti- $\gamma \mathrm{H} 2 \mathrm{AX}$ rabbit polyclonal (Upstate Biotechnology), anti-phosphorylated ATM (1981S-P) polyclonal (Bakkenist and Kastan 2003; Rockland Immunochemcial Research), or antiadenovirus 72-kD DNA-binding protein (DBP; generously provided by Dr. Peter Yacuik, St. Louis University) antibodies. In some cases, following secondary antibody staining, coverslips were washed with PBS and stained with Hoechst's dye. Staining was visualized by epifluorescence microscopy as described previously (Perez and White 1998), and percentages of cells positive for both transgene expression and or adenovirus structural proteins were determined by scoring 100-200 cells on each coverslip.

\section{RNA interference}

A total of $200 \mathrm{nM}$ of annealed, purified, and desalted doublestranded siRNA targeted against mcl-1, MCL-1 (1) (Nijhawan et al. 2003), and MCL-1 (2) (AAGGACACACAAAGCCAATGG GC; Dharmacon Research) were mixed with $175 \mu \mathrm{L}$ of OPTIMEM (Invitrogen) and incubated for $10 \mathrm{~min}$. A mixture of $3 \mu \mathrm{L}$ of Oligofectamine Reagent (Invitrogen) and $12 \mu \mathrm{L}$ of OPTI-MEM was then added to the above and incubated for $20 \mathrm{~min}$. Medium was removed from adherent HeLa cells, which were plated in DMEM without antibiotics and with $10 \%$ FBS in a 6-well dish on day 0 to achieve about $30 \%-50 \%$ confluency on day 1 . A total of $800 \mu \mathrm{L}$ of antibiotic-free medium with $10 \%$ FBS was added per well and overlaid with $200 \mu \mathrm{L}$ of the siRNA mixture. Cells were incubated at $37^{\circ} \mathrm{C}$ for $4 \mathrm{~h}$, and $500 \mu \mathrm{L}$ of DMEM with $30 \%$ serum was then added to each well. Immunoprecipitations using Brca2 and BAK antibodies was performed in CHAPS buffer as described above at $24 \mathrm{~h}$ post-transfection. The BAK antibodies used were the BAK carboxy-terminal polyclonal antibody (Pharmingen), and conformation-specific BAK antibodies BAK(Ab-1) (Oncogene) and BAK G-23 (Santa Cruz Biotechnology). Western blots were performed with BAK polyclonal antibody (Upstate Biotechnology), MCL-1 polyclonal antibody (Stressgen).

\section{Acknowledgments}

We thank Dr. Deirdre Nelson for critical reading of the manuscript, Guanghua Chen for technical assistance, Drs. Jeehyeon Bae and Aaron J. Hsueh (Stanford University) for the plasmid pCDNA3-FLAG-MCL-1L, and Dr. Peter Yacuik (St. Louis University) for the anti-adenovirus DBP monoclonal antibody. This work was funded by NCI grant R37-CA53370 and the Howard Hughes Medical Institute. 
The publication costs of this article were defrayed in part by payment of page charges. This article must therefore be hereby marked "advertisement" in accordance with 18 USC section 1734 solely to indicate this fact.

\section{References}

Bae, J., Leo, C.P., Hsu, S.Y., and Hsuch, A.J. 2000. MCL-1S, a splicing variant of the antiapoptotic BCL-2 family member MCL-1, encodes a proapoptotic protein possessing only the BH3 domain. J. Biol. Chem. 275: 25255-25261.

Bakkenist, C. and Kastan, M.B. 2003. DNA damage activates ATM through intermolecular autophosphorylation and dimer dissociation. Nature 421: 499-506.

Bassing, C.H., Suh, H., Ferguson, D.O., Chua, K.F., Manis, J., Eckersdorff, M., Gleason, M., Bronson, R., Lee, C., and Alt, F.W. 2003. Histone H2AX: A dosage-dependent suppressor of oncogenic translocations and tumors. Cell 114: 359-370.

Brader, K.R., Wolf, J.K., Hung, M.C., Yu, D., Crispens, M.A., van Golen, K.L., and Price, J.E. 1997. Adenovirus E1A expression enhances the sensitivity of an ovarian cancer cell line to multiple cytotoxic agents through an apoptotic mechanism. Clin. Cancer Res. 3: 2017-2024.

Celeste, A., Diflippantonio, S., Diflippantonio, M.J., FernandezCapetillo, O., Pilch, D.R., Sedelnikova, O.A., Eckhaus, M., Ried, T., Bonner, W.M., and Nussenzweig, A. 2003. H2AX haploinsufficiency modifies genomic stability and tumor susceptibility. Cell 114: 371-383.

Cheng, E., Wei, M., Weiler, S., Flavell, R., Mak, T., Lindsten, T., and Korsmeyer, S. 2001. BCL-2, BCL- $\mathrm{X}_{\mathrm{L}}$ sequester BH3 domain-only molecules preventing BAX- and BAK-mediated mitochondrial apoptosis. Mol. Cell 8: 705-711.

Cheng, E.H., Sheiko, T.V., Fisher, J.K., Craigen, W.J., and Korsmeyer, S.J. 2003. VDAC2 inhibits BAK activation and mitochondrial apoptosis. Science 301: 513-517.

Chiou, S.-K. and White, E. 1997. p300 binding by E1A cosegregates with p53 induction but is dispensable for apoptosis. $J$. Virol. 71: 3515-3525.

Chiou, S.-K., Tseng, C.C., Rao, L., and White, E. 1994. Functional complementation of the adenovirus E1B $19 \mathrm{~K}$ protein with Bcl-2 in the inhibition of apoptosis in infected cells. $J$. Virol. 68: 6553-6566.

Cory, S. and Adams, J.M. 2002. The Bcl2 family: Regulators of the cellular life-or-death switch. Nat. Rev. Cancer 2: 647656.

Craig, R.W. 2002. MCL1 provides a window on the role of the BCL2 family in cell proliferation, differentiation and tumorigenesis. Leukemia 16: 444-454.

Croxton, R., Ma, Y., and Cress, W.D. 2002a. Differences in DNA binding properties between E2F1 and E2F4 specify repression of the Mcl-1 promoter. Oncogene 21: 1563-1570.

Croxton, R., Ma, Y., Song, L., Haura, E.B., and Cress, W.D. 2002b. Direct repression of the Mcl-1 promoter by E2F1. Oncogene 21: 1359-1369.

Cuconati, A. and White, E. 2002. Viral homologues of Bcl-2: Role of apoptosis in the regulation of virus infection. Genes \& Dev. 16: 2465-2478.

Cuconati, A., Degenhardt, K., Sundararajan, R., Anschel, A., and White, E. 2002. BAK and BAX function to limit adenovirus replication through apoptosis induction. J. Virol. 76: 45474558.

Debbas, M. and White, E. 1993. Wild-type p53 mediates apoptosis by E1A which is inhibited by E1B. Genes \& Dev. 7: 546554.

Degenhardt, K., Sundararajan, R., Lindsten, T., Thompson, C.B., and White, E. 2002. Bax and Bak independently promote cytochrome-c release from mitochondria. J. Biol. Chem. 277: 14127-14134.

Duensing, S. and Munger, K. 2002. The human papillomavirus type 16 E6 and E7 oncoproteins independently induce numerical and structural chromosome instability. Cancer Res. 62: 7075-7082.

Farrow, S.N., White, J.H.M., Martinou, I., Raven, T., Pun, K.-T., Grinham, C.J., Martinou, J.-C., and Brown, R. 1995. Cloning of a bcl-2 homologue by interaction with adenovirus E1B 19K. Nature 374: 731-733.

Grossman, S.R., Deato, M.E., Brignone, C., Chan, H.-M., Kung, A.L., Tagami, H., Nakatani, Y., and Livingston, D.M. 2003. Polyubiquitination of $\mathrm{p} 53$ by a ubiquitin ligase activity of p300. Science 300: 342-344.

Han, J., Sabbatini, P., Perez, D., Rao, L., Modha, D., and White, E. 1996. The E1B 19K protein blocks apoptosis by interacting with and inhibiting the p53-inducible and death-promoting Bax protein. Genes \& Dev. 10: 461-477.

Jones, N. and Shenk, T. 1979. An adenovirus type 5 early gene function regulates expression of other early viral genes. Proc. Natl. Acad. Sci. 76: 3665-3669.

Kasof, G.M., Rao, L., and White, E. 1998. Btf: A novel deathpromoting transcriptional repressor that interacts with Bcl-2 related proteins. Mol. Cell. Biol. 19: 4390-4404.

Korsmeyer, S.J., Wei, M.C., Saito, M., Weiler, S., Oh, K.J., and Schlesinger, P.H. 2000. Pro-apoptotic cascade activates BID, which oligomerizes BAK or BAX into pores that result in the release of cytochrome C. Cell Death Differ. 7: 1166-1173.

Kozopas, K.M., Yang, T., Buchan, H.L., Zhou, P., and Craig, R.W. 1993. MCL1, a gene expressed in programmed myeloid cell differentiation, has sequence similarity to BCL-2. Proc. Natl. Acad. Sci. 90: 3516-3520.

Lindsten, T., Ross, A.J., King, A., Zong, W.-X., Rathmell, J.C., Shiels, H.A., Ulrich, E., Waymire, K.G., Mahar, P., Frauwirth, K., et al. 2000. The combined functions of the proapoptotic Bcl-2 family members, Bak and Bax, are essential for the normal development of multiple tissues. Mol. Cell 6: $1389-1399$.

Lowe, S. and Ruley, H.E. 1993. Stabilization of the p53 tumor suppressor is induced by adenovirus-5 E1A and accompanies apoptosis. Genes \& Dev. 7: 535-545.

Lowe, S.W., Jacks, T., Housman, D.E., and Ruley, E.H. 1994. Abrogation of oncogene-associated apoptosis allows transformation of p53-deficient cells. Proc. Nat1. Acad. Sci. 91: 2026-2030.

Nijhawan, D., Fang, M., Traer, E., Zhong, Q., Gao, W., Du, F., and Wang, X. 2003. Elimination of Mcl-1 is required for the initiation of apoptosis following ultraviolet irradiation. Genes \& Dev. 17: 1475-1496.

Perez, D. and White, E. 1998. E1B 19K inhibits Fas-mediated apoptosis through FADD-dependent sequestration of FLICE. J. Cell Biol. 141: 1255-1266.

- 2000. TNF-a signals apoptosis through a Bid-dependent conformational change in Bax that is inhibited by E1B $19 \mathrm{~K}$. Mol. Cell 6: 53-63.

Pilder, S., Logan, J., and Shenk, T. 1984. Deletion of the gene encoding the adenovirus 5 early region 1B 21,000-molecular weight polypeptide leads to degradation of viral and cellular DNA. J. Virol. 52: 664-671.

Querido, E., Marcellus, R.C., Lai, A., Charbonneau, R., Teodoro, J.G., Ketner, G., and Branton, P.E. 1997. Regulation of p53 levels by the E1B 55-Kilodalton protein and E4orf6 in adenovirus-infected cells. J. Virol. 71: 3788-3798.

Querido, E., Blanchette, P., Yan, Q., Kamura, T., Morrison, M., Boivin, D., Kaelin, W., Conaway, R., Conaway, J.W., and 
Cuconati et al.

Branton, P.E. 2001. Degradation of p53 by adenovirus E4orf6 and E1B55K proteins occurs via a novel mechanism involving a Cullin-containing complex. Genes \& Dev. 15: 31043117.

Redon, C., Pilch, D., Rogakou, E., Sedelnikova, O., Newrock, K., and Bonner, W. 2002. Histone H2A variants H2AX and H2AZ. Curr. Opin. Genet. Dev. 12: 162-169.

Reynolds, J.E., Yang, T., Qian, L.P., Jenkinson, J.D., Zhou, P., Eastman, A., and Craig, R.W. 1994. MCL-1, a member of the Bcl-2 family, delays apoptosis induced by c-myc overexpression in Chinese hamster ovary cells. Cancer Res. 54: 63486352.

Rogakou, E., Pilch, D., Orr, A.H., Ivanova, V.S., and Bonner, W.M. 1998. DNA double-stranded breaks induce histone H2AX phosphorylation on serine 139. J. Biol. Chem. 273: 5858-5868.

Rogakou, E.P., Nieves-Neira, W., Boon, C., Pommier, Y., and Bonner, W.M. 2000. Initiation of DNA fragmentation during apoptosis induces phosphorylation of $\mathrm{H} 2 \mathrm{AX}$ histone at serine 139. J. Biol. Chem. 275: 9390-9395.

Sanchez-Prieto, R., Quintanilla, M., Cano, A., Leonart, M.L., Martin, P., Aynaya, A., and Ramon y Cajal, S. 1996. Carcinoma cell lines become sensitive to DNA-damaging agents by the expression of the adenovirus E1A gene. Oncogene 13: $1083-1092$.

Shao, R., Karunagaran, D., Zhou, B.P., Li, K., Lo, S.S., Deng, J., Chiao, P., and Hung, M.C. 1997. Inhibition of nuclear factor$\kappa \mathrm{B}$ activity is involved in E1A-mediated sensitization of radiation-induced apoptosis. J. Biol. Chem. 272: 32739-32742.

Steegenga, W.T., Riteco, N., Jochemsen, A.G., Fallaux, F.J., and Bos, J.L. 1998. The large E1B protein together with the E4orf6 protein target $\mathrm{p} 53$ for active degradation in adenovirus infected cells. Oncogene 16: 349-357.

Stiewe, T., Parssanedjad, K., Esche, H., Opalka, B., and Putzer, B.M. 2000. E1A overcomes the apoptosis block in BCR$\mathrm{ABL}+$

leukemia cells and renders cells susceptible to induction of apoptosis by chemotherapeutic agents. Cancer Res. 60: 3957-3964.

Sundararajan, R. and White, E. 2001. E1B 19K blocks Bax oligomerization and tumor necrosis factor $\alpha$-mediated apoptosis. J. Virol. 75: 7506-7516.

Sundararajan, R., Cuconati, A., Nelson, D., and White, E. 2001. Tumor Necrosis Factor-a induces Bax-Bak interaction and apoptosis, which is inhibited by adenovirus E1B 19K. J. Biol. Chem. 276: 45120-45127.

Viniegra, J.G., Losa, J.H., Sanchez-Arevalo, V.J., Cobo, C.P., Soria, V.M., Ramon y Cajal, S., and Sanchez-Prieto, R. 2002. Modulation of P13K/Akt pathway by Ela mediates sensitivity to cisplatin. Oncogene 21: 7131-7136.

Wang, S., Rowe, M., and Lundgren, E. 1996. Expression of the Epstein-Barr virus transforming protein LMP1 causes a rapid and transient stimulation of the Bcl-2 homologue Mcl-1 levels in B-cell lines. Cancer Res. 56: 4610-4613.

Ward, I.M. and Chen, J. 2001. Histone H2AX is phosphorylated in an ATR-dependent manner in response to replicational stress. J. Biol. Chem. 276: 47759-47762.

Wei, M., Zong, W.-X., Cheng, E., Lindsten, T., Panoutsakopoulou, V., Ross, A., Roth, K., MacGregor, G., Thompson, C., and Korsmeyer, S. 2001. Proapoptotic Bax and Bak: A requisite gateway to mitochondrial dysfunction and death. Science 292: 727-730.

White, E. 2001. Regulation of the cell cycle and apoptosis by the oncogenes of adenovirus. Oncogene 20: 7836-7846.

White, E., Grodzicker, T., and Stillman, B.W. 1984. Mutations in the gene encoding the adenovirus E1B 19K tumor antigen cause degradation of chromosomal DNA. J. Virol. 52: 410419.

Zong, W.-X., Lindsten, T., Ross, A.J., MacGregor, G.R., and Thompson, C.B. 2001. BH3-only proteins that bind pro-survival Bcl-2 family members fail to induce apoptosis in the absence of Bax and Bak. Genes \& Dev. 15: 1481-1486. 


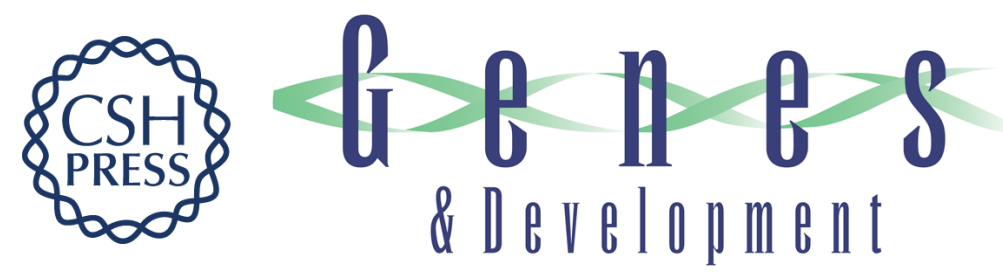

\section{DNA damage response and MCL-1 destruction initiate apoptosis in adenovirus-infected cells}

Andrea Cuconati, Chandreyee Mukherjee, Denise Perez, et al.

Genes Dev. 2003, 17:

Access the most recent version at doi:10.1101/gad.1156903

References This article cites 51 articles, 33 of which can be accessed free at: http://genesdev.cshlp.org/content/17/23/2922.full.html\#ref-list-1

License

Email Alerting

Receive free email alerts when new articles cite this article - sign up in the box at the top Service right corner of the article or click here.

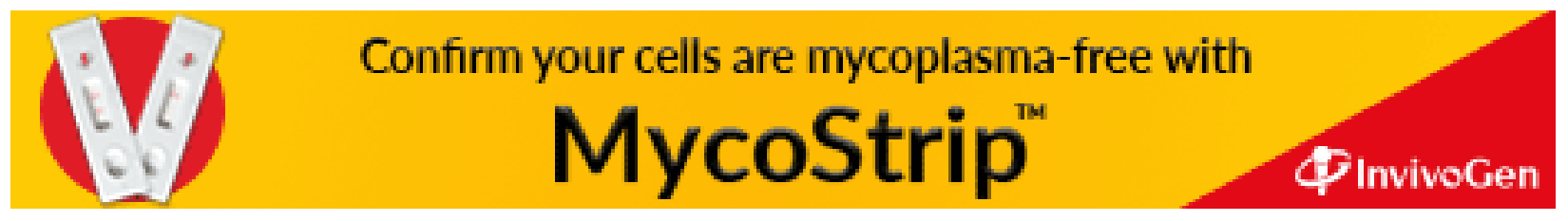

